Original article

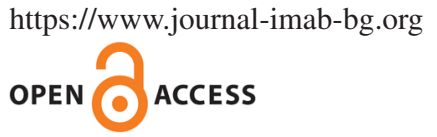

\title{
HIGH SEROPREVALENCE FOR TOXOCARIASIS AMONG MINORITY GROUPS OF VARNA RE- GION, BULGARIA
}

\author{
Tatyana Cvetkova ${ }^{1}$, Kalina Stoyanova ${ }^{1}$, Tsonko Paunov ${ }^{2}$ \\ 1) Department of Infectious Diseases, Parasitology and Dermatovenereology, \\ Faculty of Medicine, Medical University - Varna, Bulgaria. \\ 2) Department of Hygiene and Epidemiology, Faculty of Public Health, Medi- \\ cal University - Varna, Bulgaria.
}

\section{ABSTRACT:}

Toxocariasis in humans is a little-studied zoonosis, and there are no data on the impact of this parasitosis on the minority groups in our country.

The purpose of this study is to establish the seroprevalence of toxocariasis among ethnic groups in the Varna region, North-eastern Bulgaria, and compare the results with a control group from the general population.

Material/Methods: As a part of a large seroepidemiologic study, we assessed the presence of Toxocara IgG among 132 individuals, self-identified with Turkish and Roma ethnicities. As a control group, we used 250 sera from children and adults from a control group. The statistical comparison was performed regarding age, sex, and type of residence.

Results: Toxocara IgG were established in 43 individuals from the minority groups (seroprevalence of $32.58 \%$ ) and 39 persons in the control group (15.60\%) with à statistically significant difference (odds ratio $=2.61$ ). Considerably high seropositivity in the investigated ethnicities was discovered after separating by age: children-33.33\%; adults-32.00\% and gender: male-38.98\%; female-27.40\%. A detailed analysis revealed that the type of settlement is an important confounding factor. The seropositivity among the minority ethnic groups residing in small towns and villages is $35,40 \%$, in contrast to the residents of Varna city of the same ethnical origin $(15.79 \%)$; $\mathrm{OR}=2.92$.

Conclusion: The established high seroprevalence for Toxocara IgG among minority ethnic groups, especially those residing in the rural settlements of North-eastern Bulgaria, shows undiscovered to this point increased risk for the spread of toxocariasis among this population.

Keywords: Toxocariasis, Zoonosis, Seroepidemiologic Studies, Ethnic Groups, Age Distribution, Sex Distribution, Residence Characteristics,

\section{INTRODUCTION:}

Human toxocariasis is caused by the larvae of ascarid roundworms of dogs and cats - Toxocara canis and Toxocara cati. The infection occurs after the ingestion of em- bryonated ova from the environment and less common through the consumption of tissue larvae from parasitized meat of paratenic hosts. The clinical symptoms of human toxocariasis include a wide variety of clinical and laboratory findings usually combined in four specified syndromes: visceral larva migrans, ocular toxocariasis, neurotoxocariasis and covert toxocariasis [1].

The distribution of toxocariasis is ubiquitous and is directly dependent on the control of the population of the animal hosts and the pollution of the environment with infective eggs $[1,2]$. Other risk factors determining a higher risk of infection are low socioeconomic status, poor living conditions, insufficient education, and healthcare, usually associated with living in smaller settlements and rural areas $[1,3]$. Toxocariasis is one of the most spread zoonoses in developed countries. Regional studies in Europe reveal seroprevalence from $2.4 \%$ in Denmark to $31 \%$ in Ireland [1]. In Eastern Europe, the levels for specific Toxocara IgG varies between $10 \%$ and $32 \%$ [4]. Usually, higher seroprevalence is discovered in epidemiological studies originating from developing tropical countries, rural regions or communities living in low socioeconomic conditions $[1,3,5]$.

Few studies are focused on the distribution of toxocariasis in the different ethnicities and minority groups. In the USA, the seroprevalence is estimated at $21.2 \%$ in non-Hispanic blacks, $12.0 \%$ in non-Hispanic whites, and $10.7 \%$ in Mexican Americans [6]. High levels of infection (32\%) are registered in poor Andean communities of Peru [7]. Surveys among the Roma population group in Slovakia found a seroprevalence of $22.1 \%$ [5] and alarmingly high levels $(40.3 \%)$ in children of this ethnic group [8].

In our country so far, no studies have been conducted on the seroprevalence of toxocariasis among Bulgarians of Turkish and Roma ethnicity, who are the most common minority groups. According to the National Statistical Institute, the allocation of those ethnic communities in different types of settlements is immensely uneven. $77.5 \%$ of the population with Bulgarian ethnicity live in the cities, and only $22.5 \%$ live in the villages. As a contrast, almost two-thirds of those who identified themselves 
with Turkish background (62.4\%) live in rural areas, as did nearly half $(44.6 \%)$ of the Roma minorities [9]. However, in urban and rural areas, the Roma population is concentrated in isolated neighbourhoods, often outside the city's regulation, characterized by overcrowding, lack of appropriate housing, water, and sewerage infrastructure. Despite the overall improvement in the country in terms of poverty and social exclusion, $90.8 \%$ of Roma and $54.9 \%$ of the Turkish ethnic group still live at risk [10].

This study aims to establish the seroprevalence of toxocariasis among Turkish and Roma ethnicities in Northeastern Bulgaria and compare the results with a control group from the general population.

\section{MATERIAL AND METHODS:}

The current study is focused on determining the toxocariasis' prevalence in minority groups and represents a part of the results from a broader epidemiological survey researching the overall seroprevalence among the population of North-eastern Bulgaria. The minorities risk group comprises a total of 132 responders ( 57 children and 75 adults) tested on outpatient grounds in the course of preventive examinations, self-identified as such with Roma or Turkish ethnicity. The control group included 250 individuals ambulatory examined for prophylactic indications (100 children and 150 adults) self-identified as such with Bulgarian ethnicity. To assess the impact of the type of settlement on the risk of contracting a zoonotic disease such as toxocariasis, the results were evaluated in two separate groups - people living in the district city of Varna and people living in smaller towns and villages of the region.

Screening for specific Toxocara IgG was performed using a commercial kit for enzyme-linked immunosorbent assay (ELISA - Ridascreen R - Biopharm). Due to crossreactivity, the sera with positive or borderline levels were subjected to additional testing with Western blot (LD BIO). Both immunological tests were executed according to the manufacturer instructions.

Ethical Approval: The Research Ethics Committee of Medical University - Varna approved the protocol and design for the study (No.81/28.02.2019). All participants voluntarily and anonymously contributed to the survey. Trained medical specialists gave detailed information about the study and its procedures, and informed consent was obtained from all respondents or their parents and legal guardians. In an additional questionnaire devised for the study, the participants willingly and personally determined their ethnicity and other demographic data (age, sex, type of residency). No personal identifying data are presented in the research.

Data analysis: Only the positive results confirmed with Western blot were used to calculate the final data. The information was processed and analyzed with the R language and environment for statistical computing [11]. The detected prevalence is reported as the relative frequency within the $99.9 \%$ confidence interval (CI) estimated by Clopper-Pearson's exact method for binomial proportions. The size of the observed variations is represented by odds ratios (OR). Every OR is accompanied by its $99.9 \%$ asymptotic CI and the value of the exact probability ( $p$-value) for the OR being equal to 1 .

\section{RESULTS:}

The summarised results of the study presented as a number of responders, individuals with detected specific Toxocara $\mathrm{IgG}$, and the relative share (seroprevalence) among the minority and control group are displayed in table 1.

Table 1. Seroprevalence of toxocariasis and distribution by age and gender in the minority ethnic group and the control group.

\begin{tabular}{|c|c|c|c|c|c|c|c|}
\hline & \multicolumn{3}{|c|}{ Minorities } & \multicolumn{3}{|c|}{ Control group } & \multirow{2}{*}{$\begin{array}{c}\text { OR (Minorities / } \\
\text { Control group) } \\
\text { (min-max) }\end{array}$} \\
\hline & Tested & Positive & $\begin{array}{c}\text { Seroprevalence } \\
\%(\mathrm{CI}) \\
\end{array}$ & Tested & Positive & $\begin{array}{c}\text { Seroprevalence } \\
\%(\mathrm{CI})\end{array}$ & \\
\hline Children & 57 & 19 & $33.33 \%(21.40-47.06)$ & 100 & 19 & $19.00 \%(11.84-28.07)$ & $2.13(1.01-4.48)$ \\
\hline Adults & 75 & 24 & $32.00 \%(21.69-43.78)$ & 150 & 20 & $13.33 \%(8.34-19.84)$ & $3.00(1.53-5.89)$ \\
\hline Male & 59 & 23 & $38.98 \%(26.55-52.56)$ & 110 & 18 & $16.36 \%(10.00-24.62)$ & $3.27(1.58-6.76)$ \\
\hline Female & 73 & 20 & $27.40 \%(17.61-39.09)$ & 140 & 21 & $15.00 \%(9.53-22.01)$ & $2.14(1.07-4.27)$ \\
\hline Total & 132 & 43 & $32.58 \%(24.68-41.27)$ & 250 & 39 & $15.60 \%(11.33-20.70)$ & $2.61(1.59-4.31)$ \\
\hline
\end{tabular}

The screening for specific antibodies against Toxocara spp. among the persons of Roma and Turkish ethnicities found 43 seropositive individuals, which equals a high seroprevalence of $32.58 \%$. Among the control group of the general population, the relative share of the seropositive results was significantly lower $-15.6 \%$. The greater degree of infestation in the minorities risk group is a nota- ble finding at all levels of the demographic separation of the data - in children and adults and in males and females (table 1).

To assess the possible effect of the place of residence on the distribution of the results, the data on seropositivity was furthermore separated according to the responders' place of residence (table 2). 
Table 2. Seroprevalence of toxocariasis among the minority risk group and the control group of North-eastern Bulgaria, according to the type of residency.

\begin{tabular}{|c|c|c|c|c|c|c|c|}
\hline \multicolumn{8}{|c|}{ A) Varna city } \\
\hline & \multicolumn{3}{|c|}{ Minorities } & \multicolumn{3}{|c|}{ Control group } & \multirow{2}{*}{$\begin{array}{c}\text { OR (Minorities } / \\
\text { Control group) } \\
(\text { min-max })\end{array}$} \\
\hline & Tested & Positive & $\begin{array}{c}\text { Seroprevalence } \\
\%(\mathrm{CI})\end{array}$ & Tested & Positive & $\begin{array}{c}\text { Seroprevalence } \\
\%(\mathrm{CI})\end{array}$ & \\
\hline Children & 10 & 1 & $10.00 \%(0.25-44.50)$ & 73 & 13 & $17.80 \%(10.28-29.66)$ & $0.49(0.06-4.19)$ \\
\hline Adult & 9 & 2 & $22.22 \%(2.81-60.01)$ & 123 & 19 & $15.45 \%(8.47-21.71)$ & $1.73(0.33-9.04)$ \\
\hline Male & 7 & 1 & $14.29 \%(0.36-57.87)$ & 94 & 17 & $18.09 \%(10.90-27.37)$ & $0.75(0.09-6.69)$ \\
\hline Female & 12 & 2 & $16.67 \%(2.09-48.41)$ & 102 & 15 & $14.71 \%(8.47-23.09)$ & $1.16(0.23-5.83)$ \\
\hline Total & 19 & 3 & $15.79 \%(1.38-34.71)$ & 196 & 32 & $16.33 \%(11.44-22.26)$ & $0.96(0.26-3.49)$ \\
\hline
\end{tabular}

\section{B) Smaller settlements}

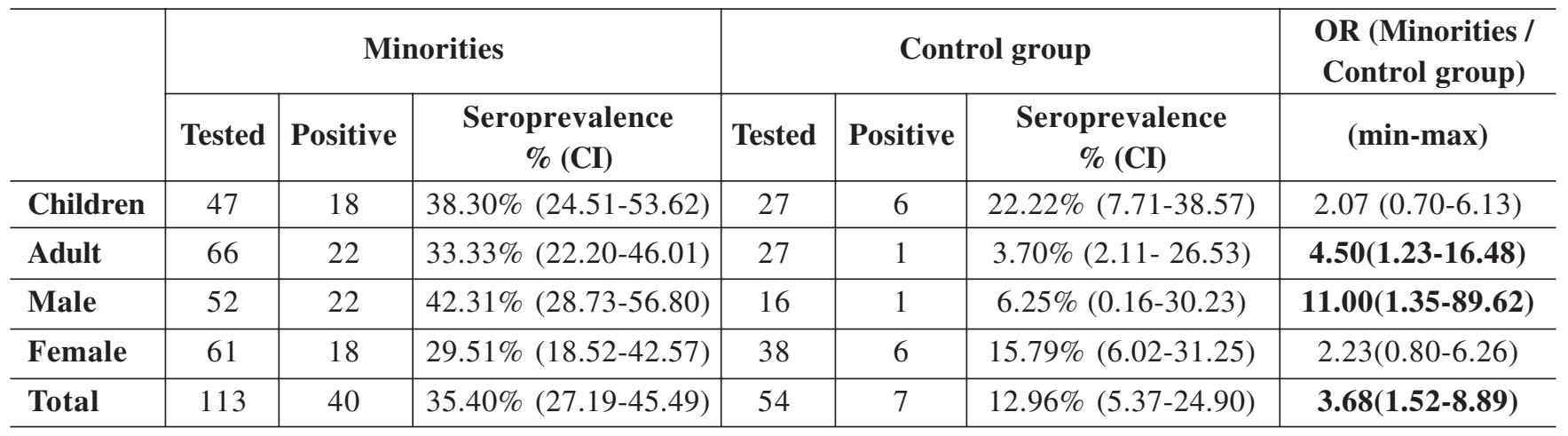

A notable finding is the lack of statistical differences in the seroprevalence in the risk group of minorities and the control group living in the city of Varna (table 2.A). In contrast, in the population of smaller settlements, a significantly higher seroprevalence among the minority risk group is established (table 2.B). This trend is permanent in both children and adults, but the highest levels of seropositivity are registered in comparison by sex with seroprevalence of $42.31 \%$ in minority males living in rural settlements.

\section{DISCUSSION:}

The distribution of toxocariasis is ubiquitous, but as any zoonosis predominant in less developed regions and among underprivileged parts of human society $[1,3]$. In Bulgaria, poverty and social exclusion are among the highest in the EU, especially in the most widespread minorities - the Roma and Turkish ethnic groups. According to the last national census, the North-eastern region has 966,097 inhabitants or $13.12 \%$ of the country's population. The most considerable fraction of the inhabitants are of Bulgarian ethnicity. The Turks are the second $(8.3 \%)$, and the Roma are the third ethnic group with a relative share of $3.6 \%$ [9]. However, the distribution of residents in the different areas of the region is exceptionally uneven. Nearly one-third of the residents $(334,870)$ are clustered in the district centre, in coherence with the countrywide trend of centralization of the population [9].
According to the lats report of the EU Agency for Fundamental Rights, the share of Roma at risk of poverty or social exclusion in Bulgaria is estimated at nearly $90 \%$ [12]. The segregation of the minorities in the small settlements leads to overcrowding, deterioration of the housing and sanitary conditions, problems with the construction and maintenance of the infrastructure, low standards of communal and personal hygiene. Still, 2/5 of the Roma in Bulgaria live in dwellings without tab water, using water from outdoor fountains or wells, 3/5 of the Roma homes are not connected to the central sewerage system, and in $4 / 5$ of the Roma dwellings, there is no indoor toilet [13]. A significant challenge in the minority population, directly resulting in poverty, is their exclusion from the labour market, consistently high levels of unemployment, and dominant engagement in low-paid occupations $[12,13]$. Another alarming and contributing factor is the educational structure of the Roma and Turkish ethnic communities. According to the 2011 census, $93 \%$ and $70 \%$ of the representatives of these ethnic groups, respectively, do not complete their secondary education, compared to about $30 \%$ for ethnic Bulgarians [9].

This first seroepidemiologic study on the prevalence of toxocariasis in North-eastern Bulgaria demonstrates the significant variances in the impact of toxocariasis among Turkish and Roma minorities (seroprevalence of $32.58 \%$ ) compared to the results in the population with Bulgarian ethnicity $(15.6 \%)$. This equals 
a statistically significant difference in the chance of infection between the two groups - OR=2.61 (1.59-4.31). Our results signify that, in fact, one-third of the minority children $(33.33 \%)$ and adults $(32.00 \%)$ are affected by this zoonosis regardless of the presence or absence of clinical symptoms. These findings are comparable with the results of similar seroepidemiologic studies among the Roma minority groups in Slovakia [5,8], which testify that the problem is relevant in other Eastern European countries as ours.

The detailed interpretation of the results found an intriguing outcome - the belonging to a minority group is not a sole determining factor for a higher chance of infection, because in the comparison of the results between the minorities and the control group living in the urban setting of Varna city neither higher seroprevalence nor significant differences in the odds ratios were discovered (table 2.A).

The relative share for the seroprevalence of toxocariasis in both populations living in urban conditions (minority ethnicities - $15.79 \%$; control group $16.33 \%$ ) is lower than the data for the rural settlements. These results are similar and comparable with the found seropositivity for toxocariasis of $17.35 \%$ in urban residents in a countrywide analysis for 2015-2017 [14]. The findings point that although the seropositivity is lower than the observed in the rural environment, the overall share of affected with Toxocara individuals is still a significant percentage. So even in bigger cities, there is a suitable environment for developing and sustaining pollution with Toxocara eggs, as demonstrated in our previous research $[2,15]$.

The results prove that for the enhanced impact of toxocariasis on the minority ethnicities, a dominant cofounding factor is living in the rural environment of the smaller settlements (table 2.B). The overall seroprevalence of the Turkish or Roma ethnicity population residing there is 35.40 (27.19-45.49), which equals a significantly increased chance of infection - $\mathrm{OR}=3.68$ (1.52-8.89). Higher seroprevalence is found in minority children living in smaller settlements $(38.30 \%)$, and this trend persists in adulthood $(33.33 \%)$ with significantly increased chances of infestation $(\mathrm{OR}=4.50 ; 1.23-16.48)$. The tendency is observable also in the comparison of the results by gender. Most affected in the entire researched population are the minority males living in smaller settlements, with a seroprevalence of $42.31 \%(28.73-56.80)$. This equals a more than 10 -fold higher risk of contracting toxocariasis $(\mathrm{OR}=11.00,1.35-89.62)$ than the males of the control group living in the same environmental conditions. The discovered shares of more than $1 / 3$ affected in every demographic stratum of the minorities living in rural settlements of North-eastern Bulgaria is alarmingly high. Such results for Toxocara seropositivity are mainly registered in the poorest regions of developing tropical countries [1, 4]. This is a testament to the low socio-economical health status and living conditions of the ethnic minorities and demonstrates the present and constant negative tendency for the increasing inequalities between the population living in urban and rural settlements.

An additional and considerable array of parasiteecosystem factors are also dominant in the rural settlements leading to enhanced and constant exposure to the animals or polluted environment. The everyday coexistence with Toxocara hosts as indoor or outdoor pets, the lack of veterinary control over the reproduction and deworming of those animals in combination with the abundant population of stray animals (dogs and cats) increase the environmental parasite load in those regions [16]. For the population in the rural regions, an existent occupational risk can be attributed to the engagement in professions associated with regular contact with hosts or polluted soil and water. Employment in the sectors of animal husbandry, farming, hunting, construction, garbage, and sewage disposal is prevalent in the rural regions, and some of these sectors mainly engage persons from minority groups $[13,15]$. Usually, the male gender dominates the majority of the listed professions, which explains the observed highest prevalence of toxocariasis among men from minorities living in small settlements.

\section{CONCLUSION:}

Human toxocariasis and its widespread distribution continue to be an unknown problem for public health and neglected infection by both veterinarian and human healthcare authorities globally. The results of our seroepidemiological study confirm that this parasitosis is one of the most common zoonotic diseases in Bulgaria, with an overall relative share above $15 \%$ in all strata of the population. The higher seroprevalence among both Turkish and Roma ethnic groups, established for the first time in this research, undoubtfully proves the existence of increased health risk for those populations. The correlation between the high seroprevalence for toxocariasis among minorities and the residency in smaller settlements corresponds to the global trends in the spread of this parasitosis. The combination of poverty, unemployment, uneducation, occupational and behavioural risk and increased environmental parasite load are well-known factors that significantly contribute to the spread of all infectious and parasitic diseases. However, their mutual potentiation in the minority groups generally segregated in the conditions of the rural environment is particularly significant for the spread of the zoonoses such as toxocariasis. Our findings strongly require increased awareness of toxocariasis as a significant public health problem and the development of focused measures for health education and veterinarian control to limit the spread of this infection and other zoonotic parasitoses minority groups and rural settlements.

\section{Acknowledgement:}

Research reported in this publication was supported by the "Science" Fund of Medical University-Varna (\#18002, Competition Session 2018). 
REFERENCES:

1. Rostami A, Ma G, Wang T, Koehler AV, Hofmann A, Chang BCH, et al. Human toxocariasis - A look at a neglected disease through an epidemiological 'prism'. Infect Genet. 2019 Oct;74:104002. [PubMed]

2. Cvetkova T, Stoyanova K, Paunov T. Contamination with Toxocara spp. eggs of environmental samples of public places of Varna city, Bulgaria. $J$ of IMAB. 2018 Jul-Sep; 24(3):217780. [Crossref]

3. Holland CV. Knowledge gaps in the epidemiology of Toxocara: the enigma remains. Parasitology. 2017 Jan;144(1):81-94. [PubMed]

4. Hotez PJ, Gurwith M. Europe's neglected infections of poverty. Int $J$ Infect Dis. 2011 Sep;15(9):611-9. [PubMed]

5. Antolova D, Jarcuska P, Janicko M, Madarasova-Geckova A, Halanova M, Cislakova L, et al. Seroprevalence of human Toxocara infections in the Roma and non-Roma populations of Eastern Slovakia: a cross-sectional study. Epidemiol Infect. $2015 \mathrm{Jul}$; 143(10):2249-58. [PubMed]

6. Liu EW, Chastain HM, Shin SH,
Wiegand RE, Kruszon-Moran D, Handali S, et al. Seroprevalence of Antibodies to Toxocara Species in the United States and Associated Risk Factors, 2011-2014. Clin Infect Dis. 2018 Jan 6;66(2):206-12. [PubMed]

7. Espinoza YA, Huapaya PE, Roldan WH, Jimenez S, Abanto EP, Rojas CA, et al. Seroprevalence of human toxocariasis in Andean communities from the Northeast of Lima, Peru. Rev Inst Med Trop Sao Paulo. 2010 Feb;52(1):31-6. [Crossref]

8. Feckova M, Antolova D, Zaleœny G, Halanova M, Strkolcova G, Weissova $\mathrm{T}$, et al. Seroepidemiology of human toxocariasis in selected population groups in Slovakia: A cross-sectional study. J Infect Public Health. 2020 Aug;13(8):1107-11. [PubMed]

9. 2011 Population census in the Republic of Bulgaria. National Statistical Institute of Bulgaria. 2012 [nternet]

10. Situational analysis for children and women in Bulgaria. UNICEF. 2018. [nternet]

11. R Core Team. R: A Language and Environment for Statistical Com- puting. R Foundation for Statistical Computing, Vienna. 2018. [Internet]

12. European Union Agency for Fundamental Rights. Second European Union minorities and discrimination survey: main results. Luxembourg: Publications Office of the European Union. 2017. p.52. [Internet]

13. [National Strategy of the Republic of Bulgaria for Roma Integration (2012 - 2020)] [in Bulgarian] Council of Ministers. Portal for public consultations. 1 March 2012. [Internet]

14. Harizanov R, Rainova I, Kaftandjiev I. Human Cystic echinococcosis, Trichinellosis and Toxocariasis in Bulgaria: an update of data for 2015-2017. Int Med. 2019; 1(2):4350. [Internet]

15. Cvetkova T, Stoyanova K. [Toxocara seroprevalence among risk group for zoonotic distribution and healthy adults.] [in Bulgarian] Varna Med Forum. 2017 Nov;6(Suppl 2):496502. [Internet]

16. Uzunova K, Nikolova N. [Etiological aspects of the stray dog's population in Bulgaria.] [in Bulgarian] Vet Assem. 2017; 1-2:41-4. [Internet]

Please cite this article as: Cvetkova T, Stoyanova K, Paunov T. High seroprevalence for toxocariasis among minority groups of Varna region, Bulgaria. J of IMAB. 2021 Jul-Sep;27(3):3858-3862.

DOI: https://doi.org/10.5272/jimab.2021273.3858

Received: 21/10/2020; Published online: 26/07/2021

\author{
Address for correspondence: \\ Kalina Stoyanova Pavlova \\ Department of Infectious Diseases, Parasitology and Dermatovenereology, Faculty \\ of Medicine, Medical University - Varna, \\ 55 Marin Drinov Str., 9000 Varna, Bulgaria \\ E-mail: drkalinasto@gmail.com
}

